\title{
Time-resolved electronic capture in germanium doped with hydrogen-like impurity centers
}

\author{
N. Deßmann ${ }^{\mathrm{a}}$, S. G. Pavlov ${ }^{\mathrm{b}}$, V. N. Shastin ${ }^{\mathrm{c}}$, R. Kh. Zhukavin ${ }^{\mathrm{c}}$, S. Winnerl ${ }^{\mathrm{d}}$, M. Mittendorff $^{\mathrm{d}}$ \\ and H.-W. Hübers ${ }^{\mathrm{a}, \mathrm{b}}$ \\ a Institut für Optik und Atomare Physik, Technische Universität Berlin, Berlin, Germany, \\ ${ }^{\mathrm{b}}$ Institute of Planetary Research, German Aerospace Center (DLR), Berlin, Germany, \\ ${ }^{c}$ Institute for Physics of Microstructures, Russian Academy of Sciences, Nizhny Novgorod, Russia, \\ ${ }^{\mathrm{d}}$ Helmholtz-Zentrum Dresden-Rossendorf, Dresden, Germany
}

\begin{abstract}
The capture of free holes and electrons in germanium (Ge) doped by gallium (Ga) or antimony (Sb) has been studied by a time-resolved pump-probe experiment with the free-electron laser FELBE at the Helmholtz-Zentrum Dresden-Rossendorf. For Ga acceptors the relaxation times decrease with increasing pump power from approximately $3 \mathrm{~ns}$ to $1 \mathrm{~ns}(2 \mathrm{~ns}$ and $1 \mathrm{~ns}$ for Sb donors, respectively). The results support the development of fast photoconductive detectors in the terahertz frequency range.
\end{abstract}

\section{INTRODUCTION AND BACKGROUND}

$\mathrm{E}$ XTRINSIC photoconductors based on Ge are very sensitive detectors in the $\mathrm{THz}$ region of the electromagnetic spectrum. The most commonly used, galliumdoped germanium (Ge:Ga), is sensitive from about 2.5 to $5 \mathrm{THz}$. It has been widely employed in space-born missions, e.g. IRAS, IRTS, ISOPHOT, Spitzer, Akari, and Herschel and is also a potential detector material for future missions such as SPICA [1].

The availability of intense but short-pulsed $\mathrm{THz}$ radiation from sources such as free electron lasers (FELs) or synchrotrons shifts the demands to broad-band detectors with very short response times. A 2-ns long decay time of the photoresponse of $\mathrm{Ge}: \mathrm{Ga}$ detectors at $2.5 \mathrm{THz}$ using highly compensated neutron transmutated Ge has been reported in [2]. The temporal response as a function of bias voltage has been investigated at $2.85 \mathrm{THz}$ for several Ge:Ga samples with different compensation. The lowest value was estimated to be 4 ns [3].

The objective of this work is to investigate the decay mechanisms of excited holes in $\mathrm{Ge}: \mathrm{Ga}$ and electrons in $\mathrm{Ge}: \mathrm{Sb}$, respectively. This helps to explore the fundamental temporal limitation of $\mathrm{Ge}$ photodetectors.

\section{EXPERIMENTAL}

In order to assess the fundamental temporal limitation of Ge photodetectors, technical aspects which affect the response time have to be excluded. These are for example the influence of an electric field or the detector bias circuit on the overall temporal behavior.

To this end typical detector material has been studied in a dedicated single-color pump-probe setup available at FELBE. FELBE provides bandwidth-limited Gaussian pulses with pulse lengths of about $10 \mathrm{ps}$ at a $13 \mathrm{MHz}$ repetition rate. The shortpulse, narrow-band radiation of the FEL allows studying impurity photoconductivity kinetics on a ps time scale and can, therefore, provide information important for optimizing the speed of response of extrinsic photoconductors.

For the measurements the samples were placed in a liquidHe flow cryostat equipped with diamond windows and cooled to about $5 \mathrm{~K}$. The maximum pulse energy coming from the FEL was $74 \mathrm{~nJ}$, corresponding to $960 \mathrm{~mW}$ average power. Fig. 1 shows the excitation process. We chose a frequency of the FEL radiation of $2.85 \mathrm{THz}$. This photon energy is larger than the ionization energy for both impurities and it fits to an atmospheric window.
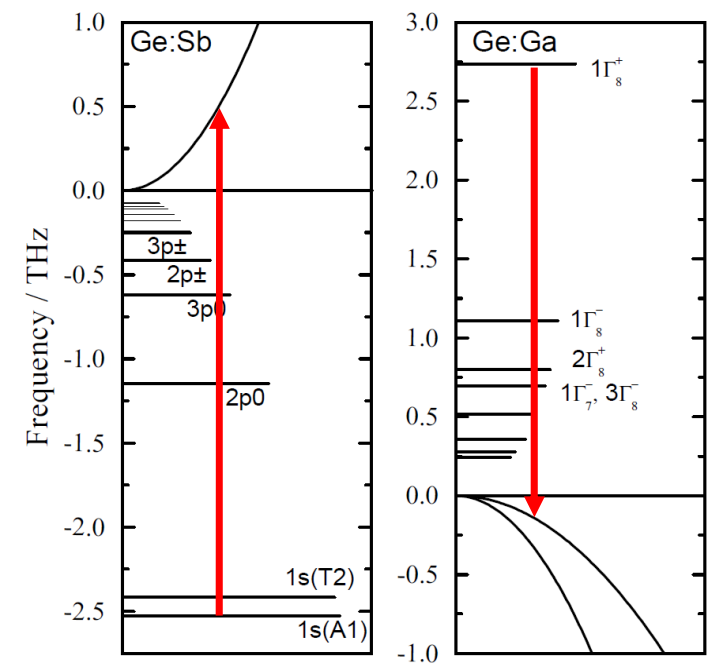

Fig. 1. Left: Energy levels of Sb and Ga impurities in germanium. The red arrow indicates the excitation by the FEL.

A p-Ge:Ga and a n-Ge:Sb sample with dimensions of $10 \mathrm{x}$ $10 \times 1 \mathrm{~mm}^{3}$ were used. The Ge:Sb sample is Czochalski-grown and the Ge:Ga sample is float zone material. The impurity concentrations are about $9 \times 10^{14} \mathrm{~cm}^{-3}(\mathrm{Ge}: \mathrm{Sb})$ and $2 \times 10^{15} \mathrm{~cm}^{-3}$ (Ge:Ga). The compensation in both samples is about $0.1 \%$.

The pump-probe technique is based on a photo-induced transmission change in the samples by a pump beam and its time-resolved measurement by a probe beam. A Mylar beam splitter divided the incoming FEL radiation into pump and probe. Both were focused onto the sample using a $10 \mathrm{~cm}$ focal length off-axis parabolic mirror. Behind the sample the transmitted pump beam was blocked whereas the probe beam was detected with a silicon bolometer. The signals were 
recorded by chopping the pump beam mechanically at about $350 \mathrm{~Hz}$ and measuring the probe signal using a lock-in amplifier.

\section{RESULTS AND DISCUSSION}

Different theoretical approaches to describe the relaxation process of ionized impurity centers in Ge can be found in the literature [4, 5]. However, no final evidence exists, that supports one model against the other. Our analysis is based on a rate equation model proposed by Bratt [6]. Neglecting effects such as radiative and Auger recombination and generation yields

$$
\frac{d n}{d t}=g-B_{T} n\left(N_{A}+n\right)
$$

for $\mathrm{n}$-type impurity centers. Where $\mathrm{g}$ is the generation rate, $\mathrm{n}$ is the number of ionized impurity centers, $\mathrm{N}_{\mathrm{A}}$ is the number of compensating acceptors and $\mathrm{B}_{\mathrm{T}}$ is the recombination rate wherein the excess carrier energy is dissipated via phonons. The interpretation of (1) gives a decay with the recombination time

$$
\tau=\frac{1}{B_{T} n\left(N_{A}+n\right)},
$$

which is inversely proportional to the number of ionized impurity centers. For a pump probe experiment, this can be attributed for by varying the energy of the pump pulse. We therefore performed the measurements for different pump powers until the probe signal was too weak to be detected.

In order to extract the time constants of the relaxation process the obtained data with one or two exponentially decaying probe transmission signals. Fig. 2 shows a typical pump-probe signal fitted by the described procedure. As Eq. (2) predicts, we observed the decay time of the signal to decrease while the pump power increased (Fig. 2). Good fits require two exponentially decaying functions with two different time constants. The presence of two time constants indicates that the decay process involves three levels.
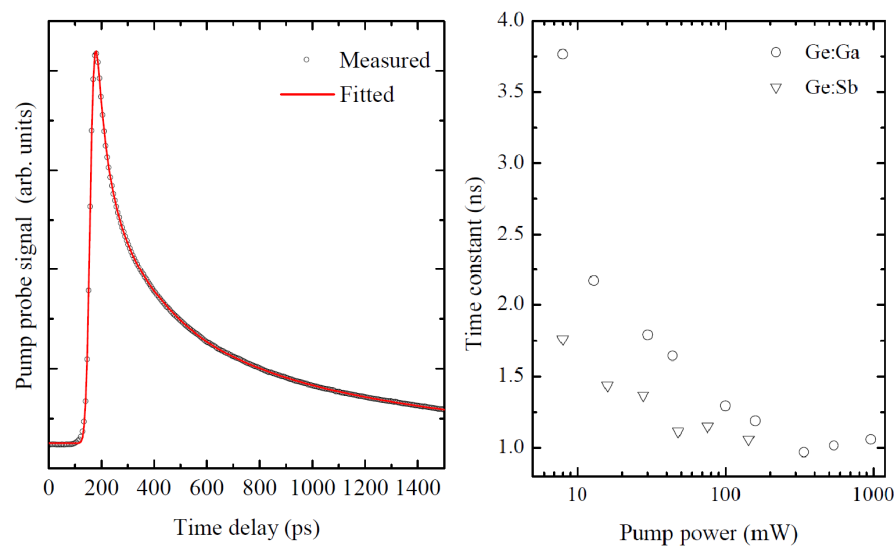

Fig. 2. Left: Typical pump-probe signal for Ge:Ga fitted as described in the text. Right: Dependences of the decay time on the pump power for Ge:Ga and Ge:Sb samples.

\section{CONCLUSION}

The decay times of photo-excited carriers in Ge doped with $\mathrm{Ga}$ or $\mathrm{Sb}$ have been determined. For both centers the decay time was found to be dependent on the average power of the pump beam. A rate equation model describes the observed decrease of the decay time of the probe signal. Deviations from a single exponential indicate the contribution of different relaxation processes, presumably three levels are involved. The presented results show that fast extrinsic Ge photoconductive detectors with a time constant as low as $1 \mathrm{~ns}$ are feasible.

\section{ACKNOWLEDGMENT}

This work was funded by the German Federal Ministry of Education and Research Grant No. 05K10KTD.

\section{REFERENCES}

[1] S. Kamiya et al., "Development of a far-infrared Ge:Ga monolithic array detector for SPICA (Space Infrared Telescope for Cosmology and Astrophysics)," pp. 5-6, 2010.

[2] F. A. Hegmann et al., "Time-resolved photoresponse of a gallium-doped germanium photoconductor using a variable pulse-width terahertz source," Applied Physics Letters, vol. 76, no. 3, p. 262, 2000.

[3] S. V. Morozov et al., "Kinetics of terahertz photoconductivity in p-Ge under impurity breakdown conditions," Semiconductors, vol. 44, no. 11, pp. 1476-1479, 2010.

[4] M. Lax, "Cascade capture of electrons in solids," Physical Review, vol. 119 , no. 5 , p. 1502,1960 .

[5] L. S. Darken, "Theoretical model for hole capture at acceptors in germanium," Physical review letters, vol. 69, no. 19, pp. 2839-2842, 1992.

[6] P. R. Bratt, "Impurity germanium and silicon infrared detectors," Semiconductors and Semimetals, vol. 12, pp. 39-142, 1977. 